\title{
Flow Generation by Rotating Colloids in Planar Microchannels
}

\author{
Ingo O. Götze ${ }^{(a)}$ and Gerhard Gomprer ${ }^{(b)}$ \\ Theoretical Soft Matter and Biophysics, Institut für Festkörperforschung, Forschungszentrum Jülich, 52425 Jülich, \\ Germany, EU
}

PACS $47.61 .-\mathrm{k}$ - Micro- and nano- scale flow phenomena

PACS 83.10.Rs - Computer simulation of molecular and particle dynamics

PACS 82.70.Dd - Colloids

\begin{abstract}
Non-equilibrium structure formation and conversion of spinning to translational motion of magnetic colloids driven by an external rotating magnetic field in microchannels is studied by particle-based mesoscale hydrodynamics simulations. For straight channels, laning is found. In ring channels, the channel curvature breaks symmetry and leads to a net fluid transport around the annulus with the same rotational direction as the colloidal spinning direction. The dependence of the translational velocity on channel width, ring radius, colloid concentration, and thermal motion is predicted.
\end{abstract}

Externally actuated and self-propelled micro- and nanorotators show an intriguing variety of non-equilibrium ' structure formation and dynamics. Examples of such systems include super-paramagnetic colloidal particles in a ' rotating magnetic field [1,4, dipolar colloids in a rotating ' electric field [5], colloidal dimers rotated by laser tweezers [6] and biological swimmers such as Volvox algae [7. The collective behavior of rotator suspensions is governed by hydrodynamic interactions. While a single rotator cannot show any directed translational motion, in ensembles of several rotators their spinning motion leads to an enhanced translational diffusion [8]. Other interesting phenomena in suspensions of rotators are stable bound states ' of spinning Volvox algae [7, spontaneous pattern formation of spinning magnetic disks at liquid-air interfaces in , the form of rotating hexagonal crystal structures [1, and spinning colloids, placed asymmetrically in a microfluidic channel, which act as micropumps [3,4,6].

A key prerequisite for the conversion of rotational into translational motion is symmetry breaking in confinement. For a single rotating cylinder close to a planar wall (and oriented parallel to the wall), the resulting hydrodynamic forces vanish since the viscous stress is balanced by the dynamic pressure field 9]; thus, a single wall is not sufficient to generate translational motion of a cylinder (in contrast to a sphere, which experiences a force parallel to the wall

\footnotetext{
(a) E-mail: i.goetze@fz-juelich.de

(b) E-mail: g.gompper@fz-juelich.de
}

in the direction expected for a rolling motion [10]). However, in the presence of a second parallel wall, more fluid is pumped through the wide gap between cylinder and wall than through the narrow gap, causing a net flow in the channel and a reaction force parallel to the wall on the cylinder - unless it is centered between the walls [11,12. Pumps based on this principle, with a typical channel diameter in the centimeter range and a fixed position of the rotating cylinder, have been studied both experimentally and numerically [11,12. The goal of microfluidics is to work with much smaller length scales, on the order of micrometers or smaller. Indeed, micron-sized pumps employing rotating colloids have been contructed recently [3, 4, 6].

With further miniaturization of microfluidic systems, thermal fluctuations become increasingly important. Furthermore, a sufficiently large concentration of particles is required to achieve reasonable flow velocities. Thus, we investigate a system of spinning colloidal particles, which are free to move in a channel, are small enough to display thermal Brownian motion, and have a finite concentration. Pairs of co-rotating cylinders in an unbounded viscous fluid are known to mutually exert forces on each other that are perpendicular to their connecting line [13], which implies a circling motion. At higher concentrations, coordinated motion occurs [8].

In a channel, many-body effects and the presence of the walls modify the interactions, and the situation becomes 

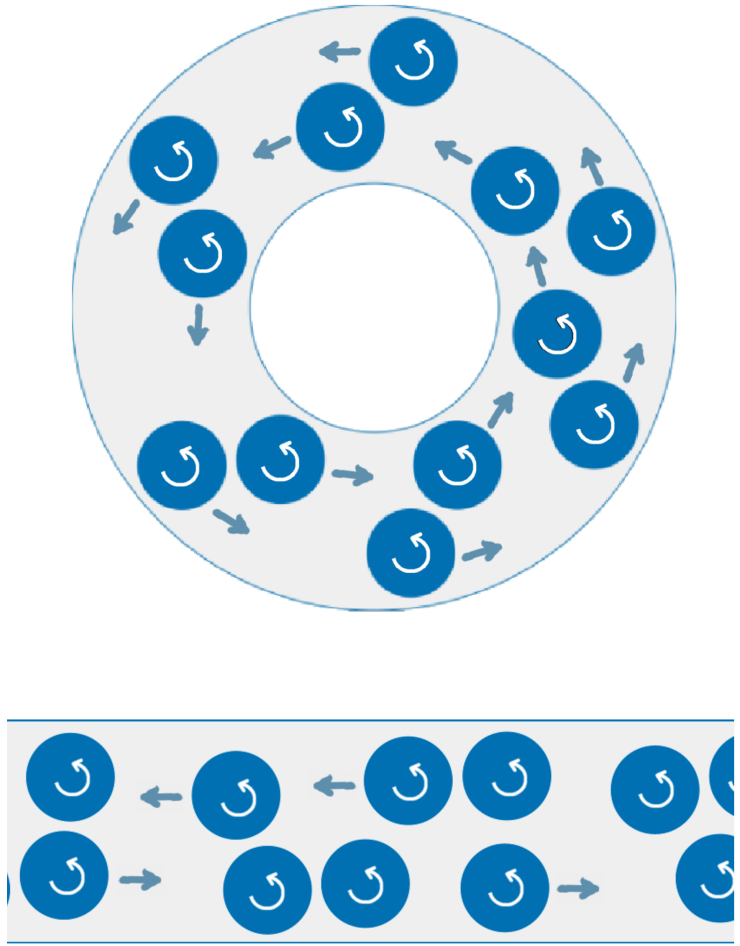

Fig. 1: (Color online) Simulation snapshots of rotating colloids in a ring channel (upper part) with median radius $R=2.4 \sigma$ and width $D=2.0 \sigma$, where $\sigma$ is the colloid diameter, and of a section of a linear channel (lower part) with $D=2.5 \sigma$. The rotational and translational motion of the colloids is illustrated by the arrows. For movies, see also footnote 1 .

much more complex. Symmetry implies that there cannot be any net fluid or colloid transport in a straight channel. However, we find that the particle distribution across the channel causes local directed motion and leads to lane formation. In ring channels, the additional symmetry breaking leads to a net translational motion along the channel, as illustrated in Fig. 1. Simulation animations of spinning colloids in a linear channel (linear_channel.avi) in a circular channel (ring_channel.avi) are available in the online supporting material. 11 We address the fundamental question how symmetry breaking due to channel curvature generates net transport in the presence of thermal fluctuations. For simplicity, we restrict ourselves to two-dimensional systems.

The fluid is modeled by multi-particle collision dynamics (MPC), a mesoscale hydrodynamics simulation technique that naturally includes thermal fluctuations [14-16].

1 The movie ring-channel. avi shows a simulation animation of $2 \mathrm{D}$ colloids in a ring channel of median radius $R=2.4 \sigma$ and width $D=2.0 \sigma$. The resulting fluid velocity field is displayed by arrows. The movie linear_channel. avi shows counter-clockwise rotating $2 \mathrm{D}$ colloids in a linear channel of width $D=2.5 \sigma$. In the displayed system, the colloid area fraction is $\Phi=0.38$. In both movies, a constant external torque $L_{\text {ext }}=150 k_{B} T$ is applied.

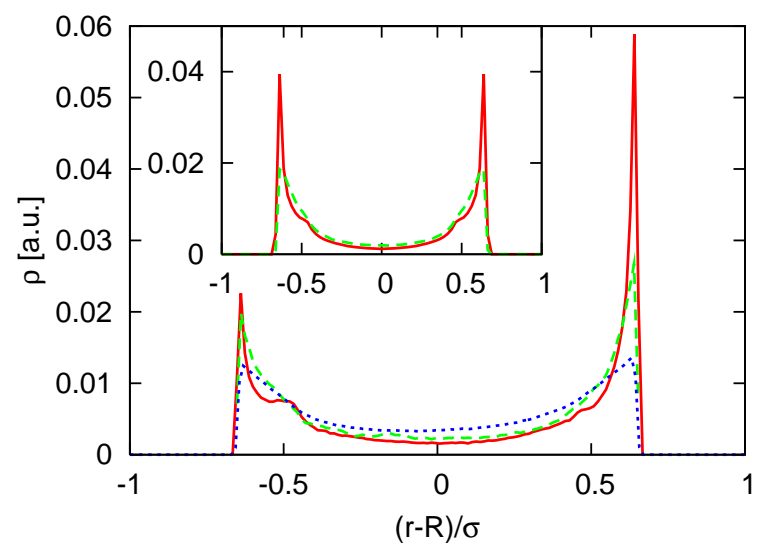

Fig. 2: (Color online) Colloid density distribution across a ring channel of median radius $R=2.4 \sigma$ and width $D=2.5 \sigma$, with area fraction $\Phi=0.25$. Data are shown for torques $L_{\text {ext }, 0}$ (full red line) and $L_{\text {ext, } 0} / 3$ (dashed green line), as well as for colloids at thermal equilibrium (dotted blue line). Inset: Same for a straight channel with torques $L_{\text {ext }, 0}$ (full red line) and $L_{\text {ext }, 0} / 9$ (dashed green line).

Here, the fluid is represented by point particles; their dynamics proceeds in two alternating steps. In the streaming step, the fluid particles move ballistically. Subsequently, they are sorted into the cells of a square lattice with lattice constant $a$. New relative velocities (with respect to the center-of-mass velocity of each cell) are assigned to all fluid particles in a collision cell, which mimics the simultaneous interaction of these particles. The algorithm is constructed such that linear momentum is conserved in each cell, in order to obtain correct hydrodynamic behavior. For a study of hydrodynamics of rotating colloids with fixed torque, it is essential to employ a variant of MPC which also conserves angular momentum [17 19.

The colloids are modeled as discs of diameter $\sigma$ with noslip boundary conditions. For the colloid-colloid and the colloid-wall interactions, a purely repulsive (shifted truncated) Lennard-Jones (LJ) potential is added. Its range $\delta_{\mathrm{LJ}} \approx 0.1 \sigma$ is chosen to be small compared to the colloid radius, but large enough to ensure that there is at least one collision box of fluid between surfaces. This guarantees that hydrodynamic interactions are captured quantitatively even at the smallest colloid distances [18. We apply a constant external torque $L_{\text {ext }}$ to all colloids, causing the colloids to spin counter-clockwise about their axes with an average angular velocity $\Omega_{\infty}=L_{\text {ext }} /\left(\eta \pi \sigma^{2}\right)$ in the bulk, where $\eta$ is the viscosity of the fluid. In a channel, the colloids develop a characteristic translational velocity $v_{0}$. The Péclet number for the rotating colloids is then defined by $\mathrm{Pe}=\sigma v_{0} / D_{t}$, where $D_{t}$ is the (geometry-dependent) translational diffusion coefficient. The Péclet number depends both on the channel geometry and the external torque. For the largest torque considered, $L_{\text {ext }, 0}=150 k_{B} T$, the Péclet number varies between 

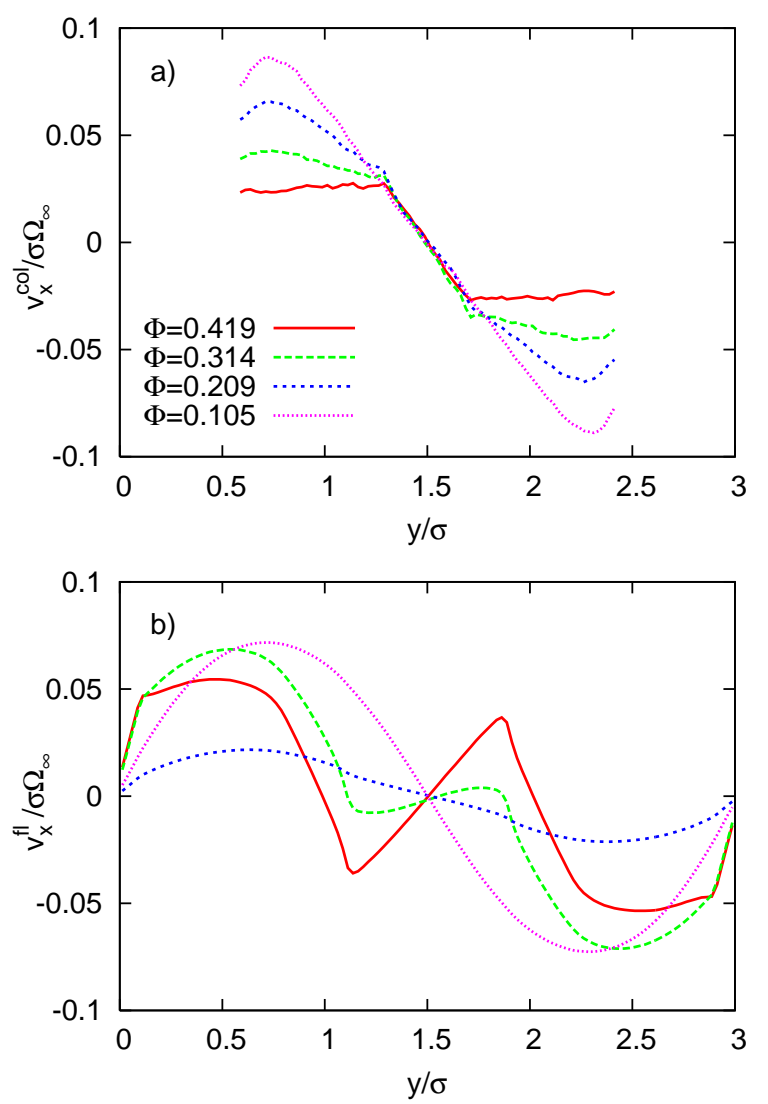

Fig. 3: (Color online) (a) Colloid velocity profiles for various colloid area fractions $\Phi$ for a straight channel of width $D=3 \sigma$. (b) The corresponding fluid velocity profiles.

$\mathrm{Pe}=\mathcal{O}(10)$ (narrowest channel) and $\mathrm{Pe}=\mathcal{O}\left(10^{2}\right)$ (widest channel). For the widest channel, the Péclet number varies linearly with the colloid torque, with $\mathrm{Pe}=\mathcal{O}(10)$ for the smallest torque $L_{\text {ext }, 0} / 9=16.7 k_{B} T$. If not mentioned otherwise, the torque $L_{\text {ext }, 0}$ is employed.

First, we investigate straight channels that are wide enough to allow colloids to pass each other, with widths $D=2.5 \sigma$ and $D=3 \sigma$. We observe that the spinning colloids move in opposite directions at opposing walls (see movie linear_channel.avi), and with increasing concentration approach the walls very closely, see Fig. 2, with a distance only slightly larger than $\sigma / 2+\delta_{\mathrm{LJ}}$. A comparison with simulations for smaller spinning velocities, see Fig. 22 shows that the peaks in the density profile broaden with decreasing $L_{\text {ext }}$. A weak density increase near the walls due to the finite colloid concentration remains even in thermal equilibrium. For wider channels, the surface excess is less pronounced.

On a qualitative level, this behavior can be understood as follows. When two spinning colloids pass each other in the channel, the hydrodynamic forces mutually exerted on each other enhance this directed motion. For symmetry reasons, colloids spinning in the center of the channel cannot propel themselves forward; however, they also cause a

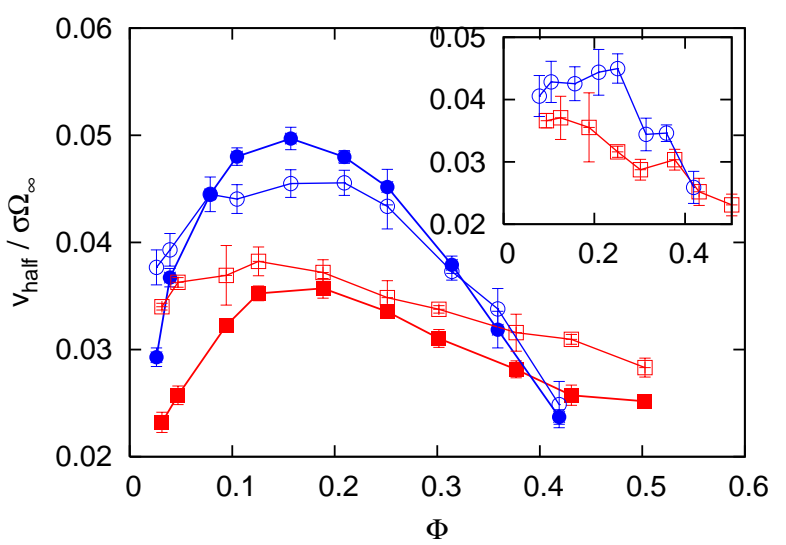

Fig. 4: (Color online) Average colloid velocity per half-channel as a function of the colloid area fraction $\Phi$ for straight channels of width $D=2.5 \sigma(\square, \boldsymbol{\square}$, red) and $D=3 \sigma(\circ, \bullet$, blue). Data are shown for three different torques, $L_{\text {ext }, 0}(\mathbf{\square}, \bullet), L_{\text {ext }, 0} / 3(\square$, o), and $L_{\text {ext,0 }} / 9$ (inset).

local fluid motion, and hence push nearby colloids towards one of the walls, while simultaneously being pushed to the opposite wall.

Colloid velocity profiles across the channel are shown in Fig. 3a for $D=3 \sigma$ and various colloid densities, together with the corresponding fluid velocity profiles in Fig. 3 b. The fluid velocity profile is a result of the combination of colloid motion pushing the fluid column ahead of it forward, the surface velocity due to colloid rotation, the no-slip boundary conditions on the walls, and the velocity field generated by the colloids near the opposite wall. Moreover, since a constant torque is exerted on the colloids, they spin faster at larger distances from the wall and at smaller (local) colloid densities. Note that the directed motion near the walls does not imply that individual colloids always move in the same direction; instead, they change lanes randomly due to thermal fluctuations and hydrodynamic interactions (which promote a circling motion of near-by colloids).

Although the fluid and colloid velocities averaged over the whole channel vanish due to symmetry reasons, there is a directed motion in both half-channels. To quantify this effect, we calculate the average colloid velocity per half-channel, as shown in Fig. 4. In the limit of vanishing colloid concentration, the only forces acting on the colloid originate from the hydrodynamic interactions with the walls. In contrast to Fig. 22 the density distribution across the channel for a single colloid is a uniform distribution for small Pe; because there is no propulsion near the channel center, but a propulsion in opposite directions near opposing walls, the velocity per half-channel approaches a finite but small value in this limit (see Fig. 4). With increasing colloid density, pairwise interactions between colloids begin to contribute. As mentioned above, when two colloids come close to each other, they circle around each other, 


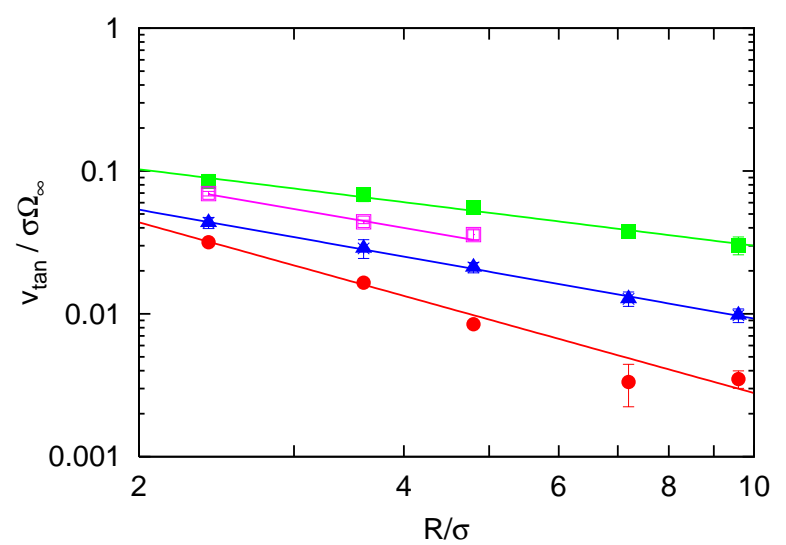

Fig. 5: (Color online) Average tangential velocity $v_{\tan }$ of all colloids as a function of the median annulus radius $R$ for curved channels with widths $D=1.5 \sigma(\bullet$, red $), 2.0 \sigma(\boldsymbol{\square}$, green), $2.5 \sigma$ $(\boldsymbol{\Lambda}$, blue) and $3.0 \sigma(\square$, magenta). The torque on each colloid is $L_{\text {ext, } 0}$.

which implies an increased and reduced density near the walls and in the center, respectively. Because the propulsion is most efficient near the walls, the colloid velocity in the half-channels increases when the colloids pass each other, and the fluid volume each colloid has to move decreases as $1 / \Phi$, the half-channel velocity increases with $\Phi$ at low densities.

At very high colloid concentrations, the average colloid velocity also becomes small because lubrication forces between neighboring colloids and between colloids and the walls slow down the rotational motion. Hence, the halfchannel velocity exhibits a maximum velocity at a finite

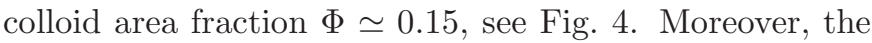
velocity is higher in the broader channel, where the colloids can pass each other more easily.

Fig. 4 also demonstrates the effect of a variation of the colloidal spinning velocity by a change of the external torque. We observe that $v_{\text {half }} /\left(\sigma \Omega_{\infty}\right)$ is nearly independent of $L_{\text {ext }}$, down to Péclet numbers as small as $\mathrm{Pe}=4.5$. In this case, diffusive motion is clearly visible, see movie (ring_lowerPE.avi) in the Supporting Material2. This implies that the directed two-lane traffic is a very robust phenomenon, which is sustainable even in the presence of large thermal noise.

Fig. 4 indicates that the colloid velocity in the zerodensity limit is lower for the highest Péclet number than for the intermediate Péclet number. We attribute this behavior to the small but finite rotational Reynolds number $\operatorname{Re}_{\text {rot }}=\rho \Omega_{\infty} \sigma^{2} /(4 \eta)$, which increases with increasing torque (where $\rho$ is the fluid density) and reaches $\mathrm{Re}_{\text {rot }}=0.37$ for the largest torque; this inertia effect im-

2 The movie ring_lowerPE.avi shows a simulation animation of $2 \mathrm{D}$ colloids in a ring channel of median radius $R=2.4 \sigma$ and width $D=2.5 \sigma$. A constant external torque $L_{\text {ext }}=16.7 k_{B} T$ is applied, a factor 9 smaller than for the movie ring_channel.avi.

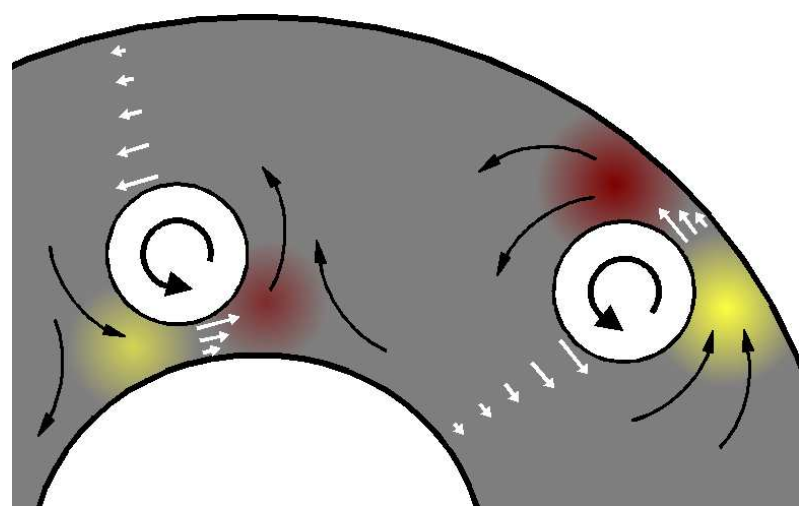

Fig. 6: (Color online) Schematic illustration of colloids spinning close to the inner and outer confining cylinders of a ring channel. Fluid is pumped towards or away from the walls (curved black arrows), giving rise to dynamic pressure. Excess pressure is indicated in yellow (light gray), low pressure in red (dark gray). The small straight white arrows depict the shear flow in the gaps.

plies a small lift force (in analogy with Refs. [1,20]) and a reduction of the colloidal density near the walls, in agreement with the simulated density profiles.

In order to obtain a net transport, symmetry breaking is necessary. The curvature of a ring channel fulfills this requirement. We characterize the geometry of the annulus by its median radius $R$ and channel width $D$. We measure the average tangential velocity $v_{\tan }$ of all colloids in the channel for various $R$ and $D$, keeping the line density $N_{\mathrm{c}} \sigma /(2 \pi R)$ fixed, where $N_{\mathrm{c}}$ is the number of colloids. Results are shown in Fig. 5 for $D=1.5 \sigma, D=2 \sigma$ and $D=2.5 \sigma$ as a function of the median channel radius $R$. We observe a total counter-clockwise tangential motion in the annulus that decreases monotonically with increasing annular radius for all investigated channel widths. Over the accessible range of annular radii, the tangential velocity $v_{\tan }$ is well described by a power-law decay,

$$
v_{\tan } \sim R^{-\gamma} .
$$

Due to the complex interplay of geometric and hydrodynamic effects, $v_{\tan }(R)$ does not obey a unique power law. For the smallest channel width, $D=1.5$, we find $\gamma \simeq 1.7$, for $D=2.0 \sigma$ an exponent $\gamma \simeq 0.76$, and for wider channels, $D=2.5 \sigma$ and $D=3.0 \sigma$, where the colloids can pass each other, $\gamma=1$. This indicates an universal exponent for $D \geq 2.5 \sigma$.

We propose that the mechanism of net colloid transport in a ring channel and its direction can be understood as follows. Consider a single colloid spinning counter-clockwise about a fixed axis close to one of the confining walls (see Fig. 6). There are three relevant contributions to the force acting on the colloid: (i) The surface motion of the colloid causes a high velocity gradient in the small gap; due to the corresponding shear stress, a force is exerted on the colloid parallel to the wall in the direction expected for a 


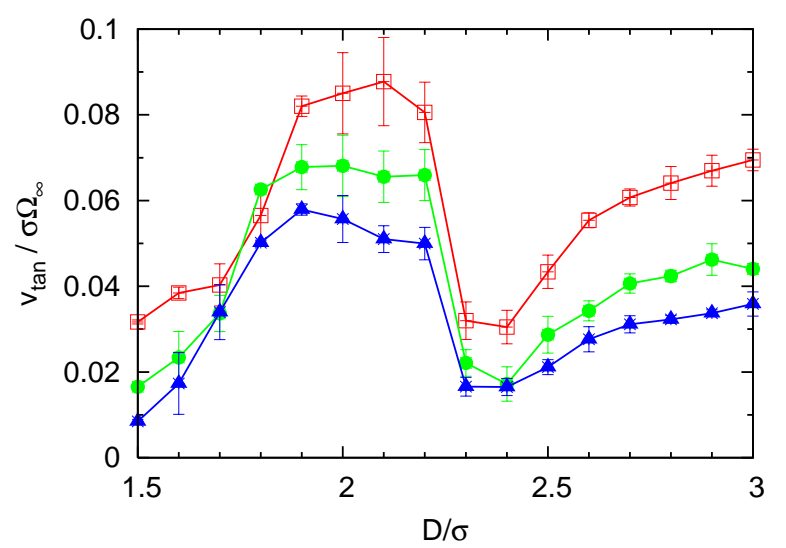

Fig. 7: (Color online) Average tangential velocity $v_{\tan }$ of the colloids as a function of channel width $D$ for fixed annulus radii $R=2.4 \sigma(\square$, red $), R=3.6 \sigma(\bullet$, green $)$ and $R=4.8 \sigma(\triangle$, blue $)$ for a constant line density $N_{c} \sigma / 2 \pi R=0.8$. The torque on each colloid is $L_{\text {ext }, 0}$.

rolling motion. (ii) A smaller velocity gradient is induced in the wide gap, causing a smaller force in the opposite direction. (iii) Fluid is pumped into or out from the small gap; the resulting pressure difference on both sides of the colloid drives it in the direction opposite to the direction expected for a rolling motion [18,21].

For a single planar wall, these three contributions cancel exactly. In case of a linear channel, the contributions (ii) and (iii) dominate over (i), causing the two way traffic. However, in a ring channel, due to the wall curvature the dynamic pressure changes compared to the linear channel. Near a convex wall, the flow caused by the spinning colloid can avoid the wall more easily than near a concave wall. Thus, the pressure gradient near the inner wall is smaller than near the outer one. Therefore, the total force acting on a colloid near the outer wall is enhanced, and the force exerted on a colloid near the inner wall is reduced. This explains the difference in the absolute value of the velocity near the confining walls that gives rise to the observed net transport in the counter-clockwise direction.

Moreover, the circumference of the outer wall is larger than of the inner wall, so that - due to geometric reasons - there are less colloids close to the inner wall that could diminish the average velocity. The latter effect becomes more important at large $D$ and small $R$, where the ratio of the circumferences of the confining walls increases.

In a ring channel, colloidal transport can also be achieved in very narrow channels, where the colloids cannot pass each other, since the forces acting on colloids near opposite walls are opposite in direction, but different in magnitude.

When the channel width $D$ is varied for a fixed median radius, the tangential velocity shows an interesting, nonmonotonic behavior, see Fig. 7 For narrow channels, the average volocity increases with increasing $D$ and reaches a maximum at $D \approx 2.1 \sigma$, where the colloids just cannot pass each other. With further increasing channel width, the average velocity drops to a minimum at $D \approx 2.4 \sigma$, and then rises again.

In the narrowest channels, with $D=1.5 \sigma$, we observe a low average tangential velocity of the colloids. One reason for this is the strong confinement that leads to a reduced spinning frequency compared to wider channels. Moreover, the colloids tend to stay close to the outer wall. This configuration allows an unhindered circulation of the fluid around the inner confining cylinder in opposite direction (compared to the motion of the colloids).

A maximum in the tangential velocity is reached in channels with $D \approx 2 \sigma$ for all investigated median radii $(2.4 \sigma<R<9.6 \sigma)$. At the maximum, because of the additional LJ potential, two colloid just cannot pass each other. Since the forces acting on colloids close to the inner and outer walls are in opposite directions, but different in magnitude, pairs of colloids become wedged together into the channel (see snapshot in Fig. 10) and perceive a net force that drives these pairs in counter-clockwise direction. As they cannot pass each other, all colloids move in the same direction. In this way, the colloids are forming plugs that the fluid can hardly pass, the fluid is efficiently dragged with these pairs, and a strong backflow of the fluid that would counteract the motion of the colloids is prevented. In the range of $D=2.0 \sigma$ to $D=2.2 \sigma$, we observe for $R=2.4 \sigma$ the formation of colloid triplets as well as pairs and combination of both (see movie ring_channel.avi), depending on the initial conditions, where both types of clusters are stable for roughly 500 full circulations around the annulus. Since all involved interactions are repulsive, the stabilization of the clusters is purely hydrodynamics. The highest tangential velocity is observed for triplets. For $R=3.6 \sigma$ and $R=4.8 \sigma$, triplets can be observed transitionally, but pairs of colloids are the preferred configuration.

In wider channels, with $D>2.2 \sigma$, where the colloids can pass each other (see movie ring_channel.avi), colloids at the inner and outer walls move in opposite directions, similar to the two-way traffic in the straight channels discussed above, and the tangential velocity drops to a minimum. However, due to a difference in the absolute values of the colloid velocities at the opposite walls, the two-way traffic is superposed with an overall translational motion and the minimum net velocity is non-zero. With further increasing channel width, the tangential velocity rises again. This observation is in accordance with the case of straight channels, where the average velocity per half-channel is higher for broader channels. Moreover, the ratio of the circumferences of the two confining cylinders increases with increasing channel width, so that there are more colloids moving in counter-clockwise direction. Because the line density is kept constant, the area density of the coloids will decrease with increasing $D$, implying that the density peaks at the walls decrease. As the transport is mainly generated near the walls, we expect the veloc- 
ity to decrease again with further increasing $D$. However, the inner confining cylinder vanishes for $D=2 R$ and the annulus loses its channel-like character well before. In the limit $D=2 R$, the ring becomes a circular cavity; in this case, colloids are known to move in counter-clockwise direction tangential to the wall, and experience a small lift force [18].

Our results in Fig. 7 can be used to estimate typical transport velocities of colloids in a ring channel. For the experimental system of Ref. [3], $\Omega=125 \mathrm{~Hz}$ and $\sigma=$ $10 \mu \mathrm{m}$, we find tangential velocities $v_{\tan }$ in the range from $10 \mu \mathrm{m} \mathrm{s}^{-1}$ to $150 \mu \mathrm{m} \mathrm{s}^{-1}$. This is more than an order of magnitude larger than the transport velocities observed for a different channel geometry in Ref. 3].

In summary, our simulations show that spinning colloids show a rich variety of non-equilibrium states in microchannels, from lane formation in straight channels to net transport in ring channels. In more complex channel networks, the fluid flow could be manipulated very easily by controlling the positions of some colloidal particles by laser tweezers, and to use them as valves or to change boundary conditions. For example, net transport in a straight channel could be induced by fixing colloidal particles on one side of the channel in regular intervals. Indeed, different kinds of boundary conditions are another interesting possibility to break the symmetry and induce flow, as has been pointed out recently for electrohydraulic pumping of dipolar molecular fluids (like water) in rotating electrical fields 22]. The manipulation of dipolar colloids by rotating electrical fields [5] is another interesting possibility, and our results should carry over to such systems to a large extent.

$$
* * *
$$

Stimulating discussions with C. Bechinger (Stuttgart) are gratefully acknowledged.

\section{REFERENCES}

[1] Grzybowski B. A., Stone H. A. and Whitesides G. M., Nature, 405 (2000) 1033.

[2] Grzybowski B. A. and Whitesides G. M., Science, 296 (2002) 718.

[3] Bleil S., Marr D. W. M. and Bechinger C., Appl. Phys. Lett. , 88 (2006) 263515.

[4] Kavčič B., Babič D., Osterman N., Podobnik B. and Poberaj I., Appl. Phys. Lett. , 95 (2009) 023504.

[5] Elsner N., Royall C. P., Vincent B. and Snoswell D. R. E., J. Chem. Phys., 130 (2009) 154901.

[6] Terray A., Oakey J. and Marr D. W. M., Science, 296 (2002) 1841.

[7] Drescher K., Leptos K. C., Tuval I., Ishikawa T., Pedley T. J. and Goldstein R. E., Phys. Rev. Lett., 102 (2009) 168101.

[8] Llopis I. and Pagonabarraga I., Eur. Phys. J. E, 26 (2008) 103.
[9] Jeffrey D. J. and Onishi Y., Q. J. Mech. appl. Math. , 34 (1981) 129.

[10] Goldman A. J., Cox R. G. and Brenner H., Chem. Eng. Sci. , 22 (1967) 637.

[11] Sen M., Wajerski D. and Gad-El-Hak M., J. Fluids Eng. , 118 (1996) 624.

[12] DeCourtye D., Sen M. and Gad-El-HaK M., Int. J. Comput. Fluid D. , 10 (1998) 13.

[13] Ueda Y., Sellier A., Kida T. and Nakanishi M., J. Fluid Mech. , 495 (2003) 225.

[14] Malevanets A. and Kapral R., J. Chem. Phys., 110 (1999) 8605.

[15] Kapral R., Adv. Chem. Phys. , 140 (2008) 89.

[16] Gompper G., Ihle T., Kroll D. M. and Winkler R. G., Adv. Polym. Sci., 221 (2009) 1.

[17] Noguchi H., Kikuchi N. and Gompper G., Europhys. Lett. , 78 (2007) 10005.

[18] Götze I. O., Noguchi H. and Gompper G., Phys. Rev. E , 76 (2007) 046705.

[19] In our MPC simulations, we employ the following parameters: density $\rho=10 a^{-2}$, collision box size $a=\sigma / 10$, time step $\Delta t=0.02 a \sqrt{m / k_{B} T}$, and fluid particle mass $m$. With these parameters, low-Reynolds-number flow is achieved in the microchannels considered here [23].

[20] Schonberg J. A. and Hinch E. J., J. Fluid Mech. , 203 (1989) 517.

[21] Müller W., Ing.-Arch. , 13 (1942) 37.

[22] Bonthuis D. J., Horinek D., Bocquet L. and Netz R. R., Phys. Rev. Lett. , 103 (2009) 144503.

[23] Ripoll M., Mussawisade K., Winkler R. G. and Gompper G., Europhys. Lett. , 68 (2004) 106. 